\title{
The Efficacy of Traditional Chinese Medicine Shoutai Pill Combined with Western Medicine in the First Trimester of Pregnancy in Women with Unexplained Recurrent Spontaneous Abortion: A Systematic Review and Meta-Analysis
}

\author{
Hui-fang Li $\mathbb{D}^{1},{ }^{1}$ Qi-hong Shen $\mathbb{D}^{2}{ }^{2}$ Xiao-qing Li, ${ }^{3}$ Zhang-feng Feng $\mathbb{D}^{4},{ }^{4}$ Wei-min Chen ${ }^{4}{ }^{4}$ \\ Jia-hua Qian, ${ }^{5}$ Li Shen, ${ }^{6}$ Li-ying Yu ${ }_{\mathbb{D}},{ }^{4}$ and Yi Yang ${ }^{1}$ \\ ${ }^{1}$ Department of TCM Gynecology, Tongxiang Maternal and Child Health-Care Center, Tongxiang, Zhejiang 314500, China \\ ${ }^{2}$ Department of Anesthesiology, Affiliated Hospital of Jiaxing University, The First Hospital of Jiaxing, Jiaxing, \\ Zhejiang 314000, China \\ ${ }^{3}$ Department of Obstetrics, Tongxiang Maternal and Child Health-Care Center, Tongxiang, Zhejiang 314500, China \\ ${ }^{4}$ Department of Gynecology, Tongxiang Maternal and Child Health-Care Center, Tongxiang, Zhejiang 314500, China \\ ${ }^{5}$ Department of Internal medicine, Tongxiang Maternal and Child Health-Care Center, Tongxiang, Zhejiang 314500, China \\ ${ }^{6}$ Department of Nursing, Tongxiang Maternal and Child Health-Care Center, Tongxiang, Zhejiang 314500, China
}

Correspondence should be addressed to Qi-hong Shen; shenqihong1989@163.com

Received 27 April 2020; Accepted 16 July 2020; Published 10 August 2020

Academic Editor: Pauline Wimberger

Copyright (C) 2020 Hui-fang Li et al. This is an open access article distributed under the Creative Commons Attribution License, which permits unrestricted use, distribution, and reproduction in any medium, provided the original work is properly cited.

\begin{abstract}
Background. Shoutai Pill (STP), a famous classic herbal formula documented in traditional Chinese medicine (TCM), is widely available in China for treating unexplained recurrent spontaneous abortion (URSA). This systematic review and meta-analysis aims at evaluating the efficacy and safety of STP in the first trimester of pregnancy in women with a history of unexplained recurrent spontaneous abortion. Methods. The following eight databases were searched from their establishment to Dec 31, 2019, for randomized controlled trials (RCTs): PubMed, Embase, Cochrane Library, Web of Science, China National Knowledge Infrastructure (CNKI), the Chinese BioMedical database (CBM), Chinese Scientific Journal Database (VIP), and the Wanfang database. The quality of evidence was estimated by the Grading of Recommendations Assessment, Development, and Evaluation (GRADE). Results. A total of 12 studies (916 patients) with URSA were contained in this meta-analysis. The forest plot showed that patients treated with Shoutai Pill and western medicine had a significantly lower incidence of early pregnancy loss (RR: 0.42; 95\% CI: $0.34-0.52 ; P<0.01, I^{2}=0 \%$ ). Subgroup analysis revealed that different types of TCM syndrome differentiation had the similar results. Also, in the combined group, patients had a lower TCM syndromes and symptoms and serum D-dimer level, while higher incidence of live birth. Conclusions. Our findings suggest that cotreatment with STP and western medicine might be superior to western medicine alone in the first trimester of pregnancy to prevent miscarriage in women with unexplained recurrent spontaneous abortion, and there was no adverse event in the experimental group reported. However, the methodological quality of included RCTs was unsatisfactory; it is necessary to verify its effectiveness with further more standardized researches of rigorous design.
\end{abstract}

\section{Introduction}

Recurrent spontaneous abortion (RSA), which is classically defined as the occurrence of two or more clinically consecutive pregnancy losses before 20 gestational weeks in fertile couples [1], is one of the most frustrating and difficult areas in reproductive medicine with complex etiologic factors varying from genetic abnormality, endocrine disorders, abnormal anatomic structures, infectious, to immune factors [2-4] and may affect about $1 \%$ to $5 \%$ of women in reproductive age 
$[3,4]$. However, approximately $50 \%$ of RSA remain unknown and unresolved [5], a condition commonly known as unexplained recurrent spontaneous abortion (URSA) after a diagnosis of exclusion and considered to be early spontaneous abortion when it occurs before 12 weeks [6]. Emerging evidence shows that immunological disturbances and abnormal coagulation and anticoagulation may be involved in this disorder $[7,8]$. Various therapeutic strategies such as immunologic intervention with allogenic lymphocyte [9] and immunoglobulin [10], anticoagulant therapy using aspirin [11] or lowmolecular weight heparin [12], hormonal supplementation with progesterone or dydrogesterone [13], and microelements supplementation, such as vitamin E, vitamin D [14], and folic acid [15], have been used to improve pregnancy outcomes among these women, but no effective treatment has been identified. To date, there is no widely accepted therapeutic approaches for URSA [16], bringing seriously physical and mental impacts to both the affected female population and their families [17-19]. Therefore, it is essential to investigate effective treatments to reduce pregnancy losses and maintain successful pregnancy preservation in patients with URSA.

In recent years, Chinese medicines have been accepted as a mainstream of medical care and have become a popular complement to western medicines in the treatment of unexplained recurrent spontaneous abortion patients, with satisfied effect and high safety and reliability [20-22]. Based on the traditional Chinese medicine theory, URSA was named as "Hua Tai" with pathogenesis being always dominated by the deficiency of the kidneys [23], which is equivalent to vital energy, stores essence, dominates reproduction, and bears a close relationship to reproductive endocrine system [24]. Patients with kidney deficiency are unable to promote blood flow leading to blood stasis and mutual regulation exists between kidney deficiency and blood coagulation [25]. Therefore, the focus of treatment is to reinforcing kidney to replenish essence, nourishing the blood and promoting blood circulation to maintain a good pregnancy [26]. Shoutai Pill (STP) is a classical herbal formula invented by a famous TCM physician Xichun Zhang in Yi Xue Zhong Zhong Can Xi Lu (Records of Tradition Chinese and Western Medicine in Combination), with the composition of four commonly used natural herbs: Chinese Dodder Seed, Himalayan Teasel Root, Chinese Taxillus Twig, and Donkey-hide Glue [27]. Over the past years, accumulating data from case reports, noncontrolled trials, animal experiments, and RCTs have generally reported consistent findings regarding the pregnancy outcome-heightening and symptom-improving effects of STP, either administrated alone or in combination with western medicine, for the management of URSA [28-30]. Despite its wide application, at present, it still lacks of comprehensive systematic review and meta-analyses to guidance. Here, we aim to investigate the efficacy and safety of TCM classical prescription Shoutai Pill combined with western medicine in patients with URSA based on a meta-analysis of the data from the selected studies.

\section{Material and Methods}

2.1. Methods. We reported this systematic review and meta-analysis following the Preferred Reporting Items for
Systematic Reviews and Meta-Analyses (PRISMA) guidelines [31]. The number of registration in PROSPERO is CRD42020169875.

2.2. Types of Studies. All of the random controlled trials (RCTs) examining the effect of Shoutai Pill combined with western medicine for the treatment of unexplained recurrent spontaneous abortion with no limitations on language and publication status were included. Non-RCTs or animal experiments were excluded.

2.3. Types of Participants. Patients who had been confirmed pregnant by serum human chorionic gonadotropin (HCG) or ultrasound in the first trimester of pregnancy with a diagnosis history of URSA, defined as two or more spontaneous abortions that had ruled out the following four definite etiologies: infections, abnormal parental karyotypes, endocrine disorders, and anatomic abnormality, regardless of maternal age, gestational age, ethnicity, nationality, education, or economic status. The inclusion criteria also stipulated that there had been no treatment given before pregnancy or the entry into the trials. Trials involving recurrent spontaneous abortion with definite etiologies and URSA participants without pregnancy were excluded.

2.4. Types of Interventions. We included studies using the common prescription of Shoutai Pill as a basic formula and modified according to syndrome differentiation meanwhile, regardless of the dose, method of dosing, or duration of administration, in combination with western medicine compared with western medicine excluding bed rest and psychological supports. The western medicine should remain the same in the control group in the same RCT. All the treatments were initiated after the patients were confirmed to be pregnant. If trials included other cointerventions such as acupuncture, acupoint application, and moxibustion, they were excluded.

2.5. Types of Outcome Measures. Primary outcome is the incidence of early pregnancy loss, as defined by the authors. Second outcomes included the incidence of live birth, as defined by the authors, TCM syndromes and symptoms, serum D-dimer level, and incidence of maternal and perinatal adverse events during treatment. Studies without pregnancy outcome reported were excluded.

2.6. Search Strategy. We systematically conducted electronic searches in the following clinical studies databases: PubMed, Embase, Cochrane Library, Web of Science, China National Knowledge Infrastructure (CNKI), the Chinese BioMedical database (CBM), Chinese Scientific Journals Database (VIP), and the Wanfang database with no limitations on language and publication status for RCTs examining the effect of Shoutai Pill combined with western medicine for the treatment of URSA from inception of each databases through to 31 December 2019. We made the retrieval formula according to the PICOS strategy. The search terms for literature searching were as follows: ("recurrent spontaneous abortion" OR "recurrent miscarriage" OR "habitual abortion" OR "recurrent pregnancy loss" OR "hua tai") AND ("shou tai pill” OR "shoutai pill” OR 


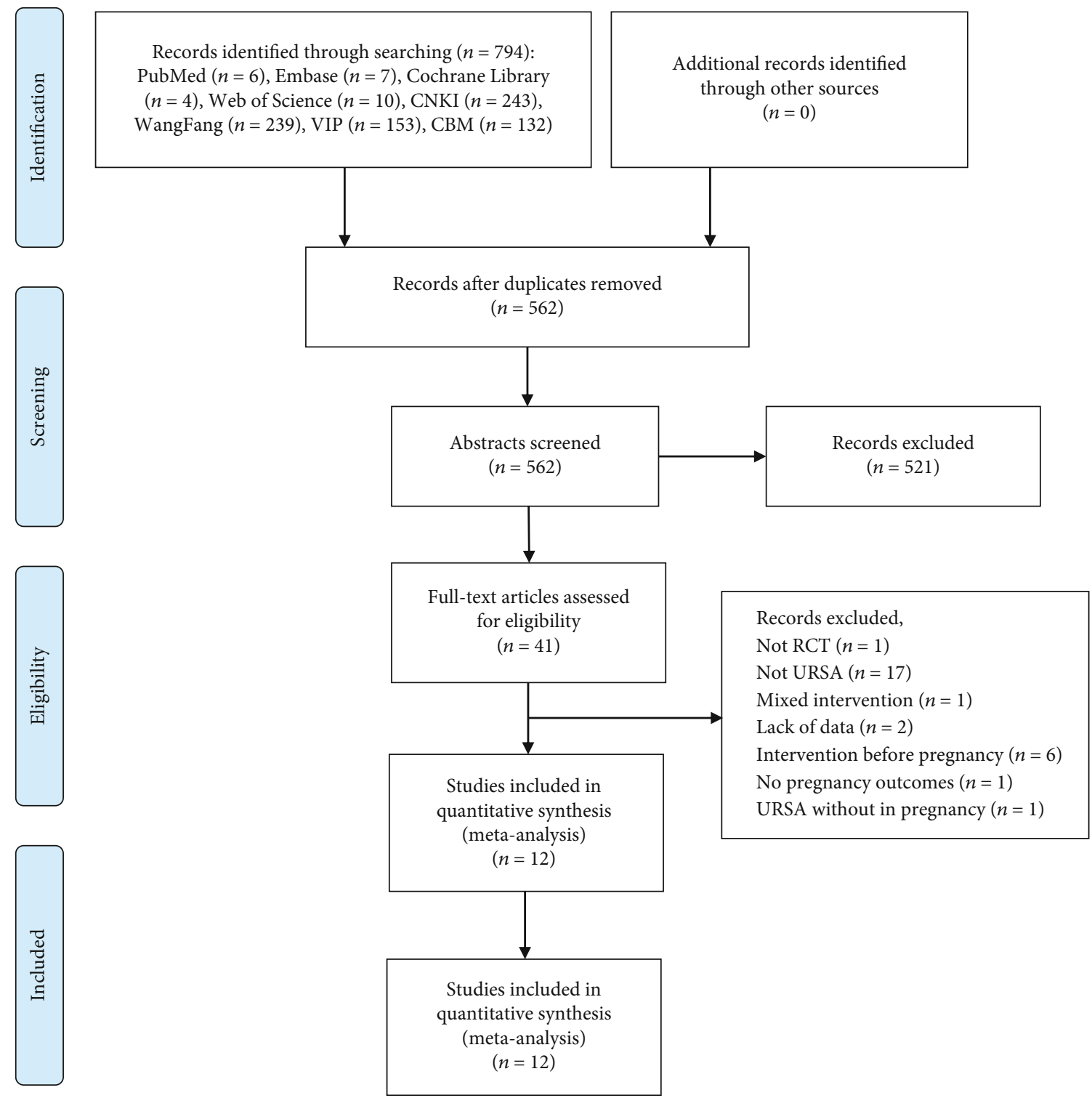

FIgURE 1: The inclusion process of literature.

"shoutai wan" OR "shou tai wan") AND ("clinical research" OR "randomized controlled trial" OR "randomization" OR "RCT" OR "random grouping"). We also manually searched the reference lists of all identified articles for possible related studies to supplement the relevant literature.

2.7. Data Extraction and Quality Assessment. Two researchers (Hui-fang Li, Qi-hong Shen) extracted the general information of the eligible studies by a predesigned and standardized data extraction form: first author, year of published, TCM syndrome differentiation, sample size, age, gestational age, times of abortions, definition of miscarriage and live birth, intervention time, treatment interventions and control groups, treatment duration, and outcomes. Any conflict was resolved by a third author (Wei-min Chen). The methodologic quality of each individual study was independently evaluated by two researchers (Li Shen, Yi Yang) in reference to the Cochrane Handbook for Systematic Reviews of
Interventions. We evaluated using the following criteria: random sequence generation, allocation concealment, blinding of participants and personnel, blinding of outcome assessments, incomplete outcome data, selective reporting, and other bias. Each study was classified into low risk, high risk, or unclear. If there was a disagreement, we referred to the views of the third researcher (Wei-min Chen).

2.8. GRADE Evaluation. The quality of outcome was evaluated by GRADE (Grading of Recommendations Assessment, Development, and Evaluation) according to the following criteria: study design, risk of bias, rating inconsistency in results, rating indirectness of evidence, and others. The quality of evidence was classified as high, moderate, low, or very low.

2.9. Statistical Analysis. We conducted this meta-analysis by using Review Manager (RevMan) (computer program) (Version 5.3. Copenhagen: The Nordic Cochrane Centre, The 


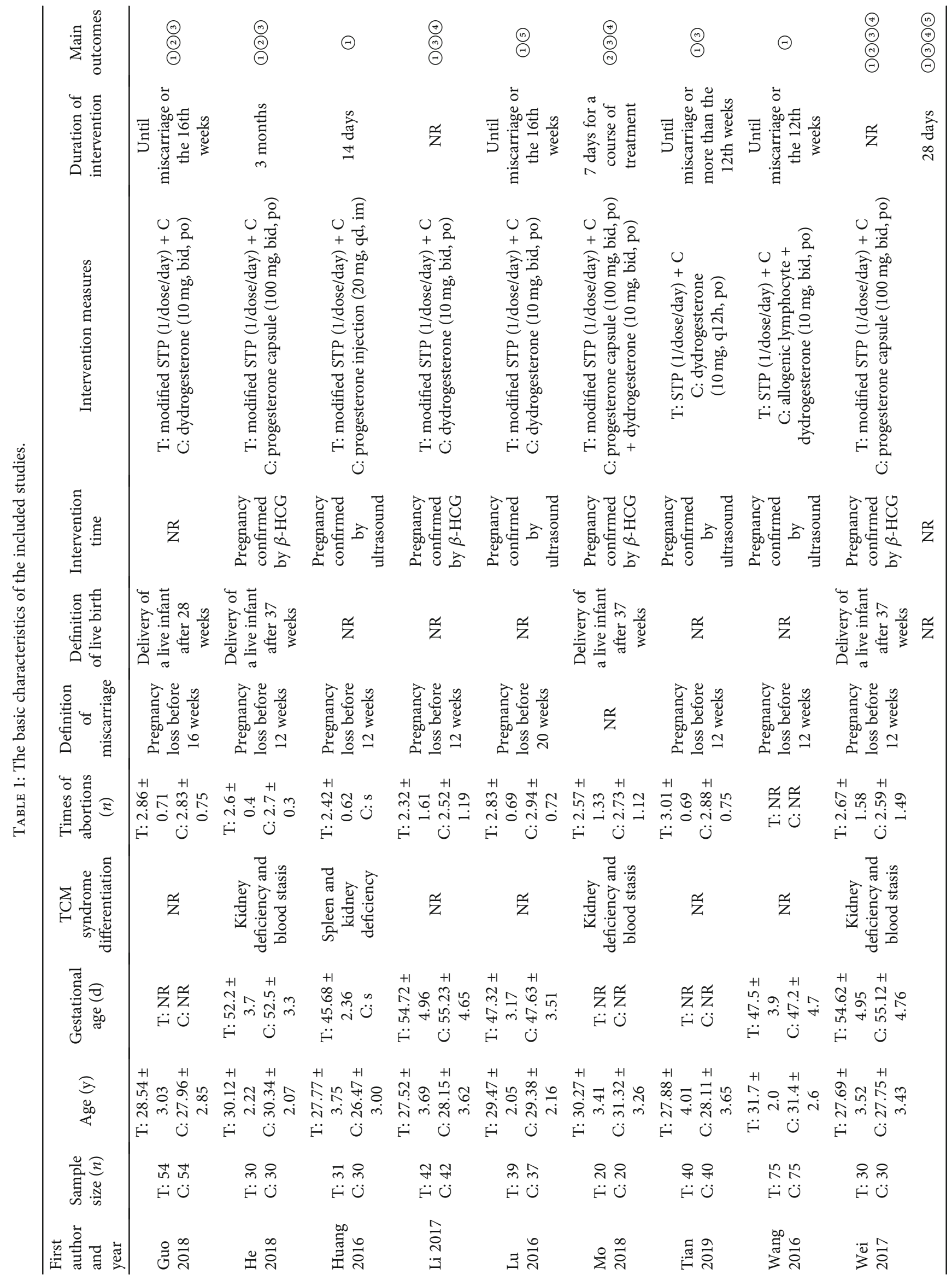




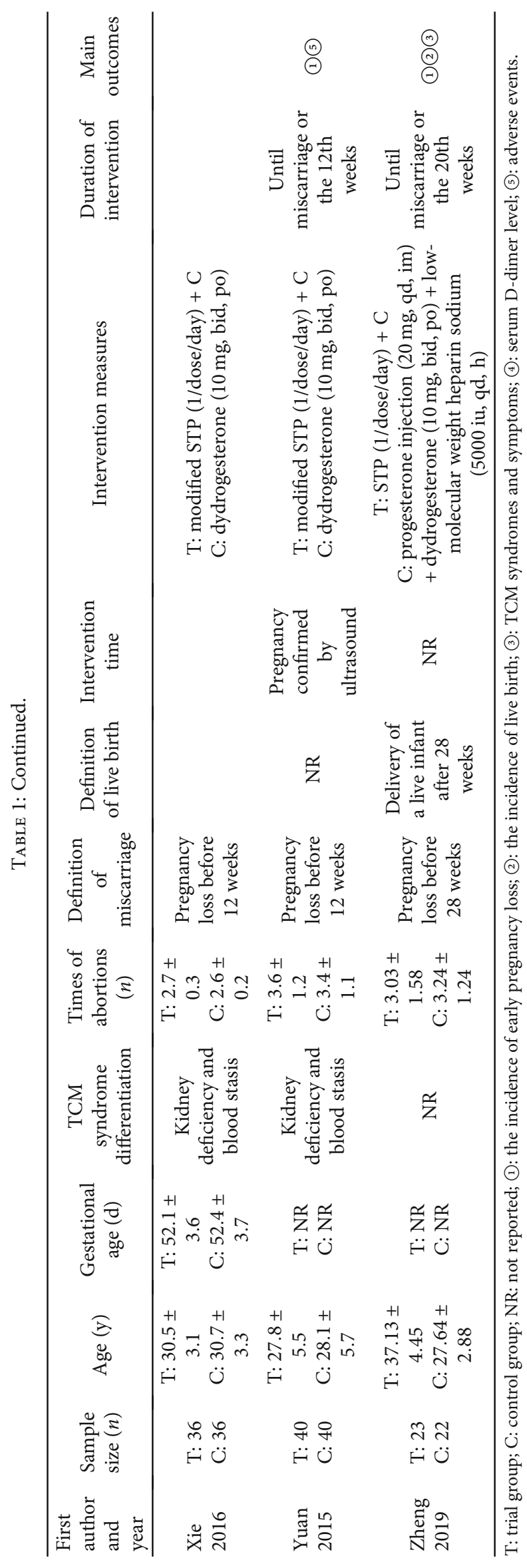


TABLE 2: Composition of prescription in the included studies.

\begin{tabular}{|c|c|c|}
\hline References & escription & Composition of prescription \\
\hline Guo et al. 2018, [32] & odified STP & $\begin{array}{c}\text { Chinese Dodder Seed } 15 \mathrm{~g} \text {, Chinese Taxillus Twig } 15 \mathrm{~g} \text {, Himalayan Teasel Root } 10 \mathrm{~g} \text {, Eucommia } \\
\text { ulmoides } 12 \mathrm{~g} \text {, Donkey-hide Glue } 12 \mathrm{~g} \text {, Cornus officinalis } 15 \mathrm{~g} \text {, Chinese wolfberry } 15 \mathrm{~g} \text {, and Liquorice } \\
\text { Root } 6 \mathrm{~g}\end{array}$ \\
\hline He et al. 2018, [33] & Modified STP & $\begin{array}{l}\text { Chinese Dodder Seed } 30 \mathrm{~g} \text {, Chinese Taxillus Twig } 30 \mathrm{~g} \text {, Himalayan Teasel Root } 20 \mathrm{~g} \text {, Eucommia } \\
\text { ulmoides } 20 \mathrm{~g} \text {, Donkey-hide Glue } 12 \mathrm{~g} \text {, Chinese Angelica } 12 \mathrm{~g} \text {, Salvia miltiorrhiza 12g, White } \\
\text { Paeony Root } 10 \mathrm{~g} \text {, Steamed Rehmannia Root } 10 \mathrm{~g} \text {, and Liquorice Root } 6 \mathrm{~g}\end{array}$ \\
\hline Huang et al. 2016, [34] & Modified STP & $\begin{array}{c}\text { Chinese Dodder Seed } 20 \mathrm{~g} \text {, Chinese Taxillus Twig } 20 \mathrm{~g} \text {, Himalayan Teasel Root } 20 \mathrm{~g} \text {, Donkey-hide } \\
\text { Glue } 10 \mathrm{~g} \text {, Radix codonopsis } 20 \mathrm{~g} \text {, Largehead Atractylodes Rhizome } 15 \mathrm{~g} \text {, Poria cocos } 10 \mathrm{~g} \text {, and } \\
\text { Liquorice root } 5 \mathrm{~g}\end{array}$ \\
\hline Li et al. 2017, [35] & Modified STP & $\begin{array}{l}\text { Chinese Dodder Seed } 30 \mathrm{~g} \text {, Chinese Taxillus Twig } 30 \mathrm{~g} \text {, Himalayan Teasel Root } 20 \mathrm{~g} \text {, Eucommia } \\
\text { ulmoides } 20 \mathrm{~g} \text {, Donkey-hide Glue } 12 \mathrm{~g} \text {, Chinese Angelica } 12 \mathrm{~g} \text {, Salvia miltiorrhiza } 12 \mathrm{~g} \text {, White } \\
\text { Paeony Root } 10 \mathrm{~g} \text {, Steamed Rehmannia Root } 10 \mathrm{~g} \text {, and Liquorice Root } 6 \mathrm{~g}\end{array}$ \\
\hline $6,[36]$ & Modified STP & $\begin{array}{c}\text { Chinese Dodder Seed } 15 \mathrm{~g} \text {, Chinese Taxillus Twig } 15 \mathrm{~g} \text {, Himalayan Teasel Root } 9 \mathrm{~g} \text {, Donkey-hide } \\
\text { Glue } 12 \mathrm{~g} \text {, Chinese Wolfberry } 15 \mathrm{~g} \text {, Cornus officinalis } 15 \mathrm{~g} \text {, Eucommia ulmoides } 12 \mathrm{~g} \text {, Salvia } \\
\text { miltiorrhiza } 12 \mathrm{~g} \text {, Cinnamomi cortex } 9 \mathrm{~g} \text {, and Liquorice Root } 6\end{array}$ \\
\hline Mo $€$ & Modifi & $\begin{array}{l}\text { Chinese Dodder Seed } 30 \mathrm{~g} \text {, Chinese Taxillus Twig } 30 \mathrm{~g} \text {, Himalayan Teasel Root } 20 \mathrm{~g} \text {, Donkey-hide } \\
\text { Glue } 12 \mathrm{~g} \text {, Eucommia ulmoides } 20 \mathrm{~g} \text {, Chinese Angelica } 12 \mathrm{~g} \text {, Salvia miltiorrhiza } 12 \mathrm{~g} \text {, White Paeony } \\
\text { Root } 10 \mathrm{~g} \text {, Steamed Rehmannia Root } 10 \mathrm{~g} \text {, and Liquorice Root } 6 \mathrm{~g}\end{array}$ \\
\hline Tian et al. 2019, [38] & STP & $\begin{array}{c}\text { Chinese Dodder Seed } 15 \mathrm{~g} \text {, Chinese Taxillus Twig } 15 \mathrm{~g} \text {, Himalayan Teasel Root } 10 \mathrm{~g} \text {, and Donkey- } \\
\text { hide Glue } 12 \mathrm{~g}\end{array}$ \\
\hline Wang et al. 2016, [42] & STP & $\begin{array}{c}\text { Chinese Dodder Seed } 40 \mathrm{~g} \text {, Chinese Taxillus Twig } 20 \mathrm{~g} \text {, Himalayan Teasel Root } 20 \mathrm{~g} \text {, and Donkey- } \\
\text { hide Glue } 20 \mathrm{~g}\end{array}$ \\
\hline Wei et al. 2017, [39] & Modified STP & $\begin{array}{l}\text { Chinese Dodder Seed } 30 \mathrm{~g} \text {, Chinese Taxillus Twig } 30 \mathrm{~g} \text {, Himalayan Teasel Root } 20 \mathrm{~g} \text {, Donkey-hide } \\
\text { Glue } 12 \mathrm{~g} \text {, Eucommia ulmoides } 20 \mathrm{~g} \text {, Chinese Angelica } 12 \mathrm{~g} \text {, Salvia miltiorrhiza } 12 \mathrm{~g} \text {, White Paeony } \\
\text { Root } 10 \mathrm{~g} \text {, Steamed Rehmannia Root } 10 \mathrm{~g} \text {, and Liquorice Root } 6 \mathrm{~g}\end{array}$ \\
\hline $016,[40]$ & Modified STP & $\begin{array}{l}\text { Chinese Dodder Seed } 30 \mathrm{~g} \text {, Chinese Taxillus Twig } 30 \mathrm{~g} \text {, Himalayan Teasel Root } 20 \mathrm{~g} \text {, Donkey-hide } \\
\text { Glue } 12 \mathrm{~g} \text {, Eucommia ulmoides } 20 \mathrm{~g} \text {, Chinese Angelica } 12 \mathrm{~g} \text {, Salvia miltiorrhiza } 12 \mathrm{~g} \text {, White Paeony } \\
\text { Root } 10 \mathrm{~g} \text {, Steamed Rehmannia Root } 10 \mathrm{~g} \text {, and Liquorice Root } 6 \mathrm{~g}\end{array}$ \\
\hline Yuan et al. 2015, [41] & Modified STP & $\begin{array}{l}\text { Chinese Dodder Seed } 30 \mathrm{~g} \text {, Chinese Taxillus Twig } 30 \mathrm{~g} \text {, Himalayan Teasel Root } 20 \mathrm{~g} \text {, Donkey-hide } \\
\text { Glue } 12 \mathrm{~g} \text {, Eucommia ulmoides } 20 \mathrm{~g} \text {, Chinese Angelica } 12 \mathrm{~g} \text {, Salvia miltiorrhiza } 12 \mathrm{~g} \text {, White Paeony } \\
\text { Root } 10 \mathrm{~g} \text {, Steamed Rehmannia Root } 10 \mathrm{~g} \text {, and Liquorice Root } 6 \mathrm{~g}\end{array}$ \\
\hline Zheng 2019, [43] & STP & $\begin{array}{c}\text { Chinese Dodder Seed } 15 \mathrm{~g} \text {, Chinese Taxillus Twig } 15 \mathrm{~g} \text {, Himalayan Teasel Root } 15 \mathrm{~g} \text {, and Root } \\
\text { Donkey-hide Glue } 15 \mathrm{~g}\end{array}$ \\
\hline
\end{tabular}

STP: Shoutai Pill.

Cochrane Collaboration, 2014). Regarding the study outcomes, relative risk (RR) with 95\% confidence interval (CI) was used for binary variables, while the weighted mean difference (WMD) and 95\% CI were presented for continuous variables. Cochrane's $P$ values and $I^{2}$ were tested to examine heterogeneity among the studies. High heterogeneity most likely existed due to the clinical and methodological factors, so the random effect model was adopted in this meta-analysis even $I^{2}$ was small. Subgroup analysis was performed based on the type of TCM syndrome: kidney deficiency and blood stasis, spleen and kidney deficiency, or without reported.

Funnel plots and the Egger regression test were performed to examine potential publish bias. In addition, sensitivity analysis was performed by sequentially deleting trials to check the stability of the primary outcomes.

\section{Result}

3.1. Search Results. Initially, a total of 794 relevant studies were collected. After excluding duplicate studies, we scanned 562 studies based on their abstracts and titles. Then, 41 arti- cles were evaluated by full text. And 29 trials were eliminated for the following reasons: seventeen non-URSA studies, one study was not RCT, one trial without pregnancy outcome reported, one study included URSA women without pregnancy, one article with mixed interventions, two articles were lacking data, and six studies giving intervention before pregnancy. Eventually, 12 studies were included in our system review. The search process is displayed in Figure 1.

3.2. Study Characteristics. The basic information of the included RCTs is summarized in Table 1. Of these trials, all of them were published in China. A total of 916 patients with URSA were contained in these studies, 460 participants were assigned to the treatment group, and 456 were designated in the control group. The sample sizes of these trials ranged from 40 to 150 . The baseline in all of the trials did not significantly differ. All of the trials involved two-arm designs: treatment group versus control group. Patients in the treatment group were treated with STP (Table 2) in combination with western medicine, while patients in the control group were administered with western medicine alone, including 
ten RCTs used natural P (progesterone injection, progesterone capsule, or dydrogesterone) [32-41], whereas the other two studies used natural $\mathrm{P}$ combined with other treatment, one with allogenic lymphocyte immunotherapy [42] and one with low-molecular weight heparin sodium [43]. Treatment duration varied from fourteen days to 20 gestational weeks with two articles not mentioned [35, 39]. Regarding TCM syndrome differentiation, 5 articles were kidney deficiency and blood stasis [33, 37, 39-41], 1 trial was spleen and kidney deficiency [34], and 6 studies not mentioned $[32,35,36,38,42,43]$. Of the 12 trials, 11 reported the incidence of early pregnancy loss [32-42], five presented the live birth rate $[32,33,37,39,43], 8$ trials mentioned the TCM syndromes and symptoms [32, 33, 35, 37-40, 43], 4 trials stated serum D-dimer level $[35,37,39,40]$, and three trials mentioned the adverse events $[36,40,41]$.

3.3. Risk of Bias Assessment. In general, the methodological quality of the included trials was poor. All of the 12 included studies involved two-arm designs and declared as random controlled trials, and 8 trials reported proper generation methods (random number table or coin toss) with a low risk of bias [32, 33, 35-37, 39-41]. Four trials did not describe the randomization procedure clearly $[34,38,42,43]$. None of the trials reported any concealed allocation or blinding of patients and investigators. No trial indicated the number and reasons of dropouts. No selective reporting was reported. None of the included trials calculated the sample size. The results of the assessments are shown in Figure 2.

\section{Outcome Measures}

4.1. Primary Outcome. Eleven studies reported the incidence of early pregnancy loss, and meta-analysis showed that patients in the combined group had a significantly lower occurrence (RR: 0.42 ; 95\% CI: $0.34-0.52 ; P<0.01, I^{2}=0 \%$, Figure 3). Subgroup analysis revealed that different types of TCM syndrome differentiation had a lower incidence of early pregnancy loss in the combined group (Figure 4).

4.2. Secondary Outcomes. Eight studies assessed TCM syndromes and symptoms, five trials reported the incidence of live birth, and four trials evaluated serum D-dimer level. Compared with the western medicine alone group, patients treated with STP and western medicine had a lower TCM syndromes and symptoms (SMD: -2.39; 95\% CI: -2.86 , -1.93; $P<0.01, I^{2}=76 \%$, Figure 5) and serum D-dimer level (SMD: $-0.25 ; 95 \%$ CI: $-0.30,-0.20 ; \quad P<0.01, I^{2}=0 \%$, Figure 6), while higher incidence of live birth (RR: 1.81; 95\% CI: $1.46-2.25 ; P<0.01, I^{2}=0 \%$, Figure 7).

In the 12 included studies, only three trials reported on adverse events. No adverse effects of STP combined with western medicine group were identified in these trials, so there was insufficient data pooled to assess the safety of this intervention.

4.3. Publication Bias and Sensitivity Analysis. Although the funnel plot for the early pregnancy loss rate was asymmetrically distributed, Egger's test showed no potential publish

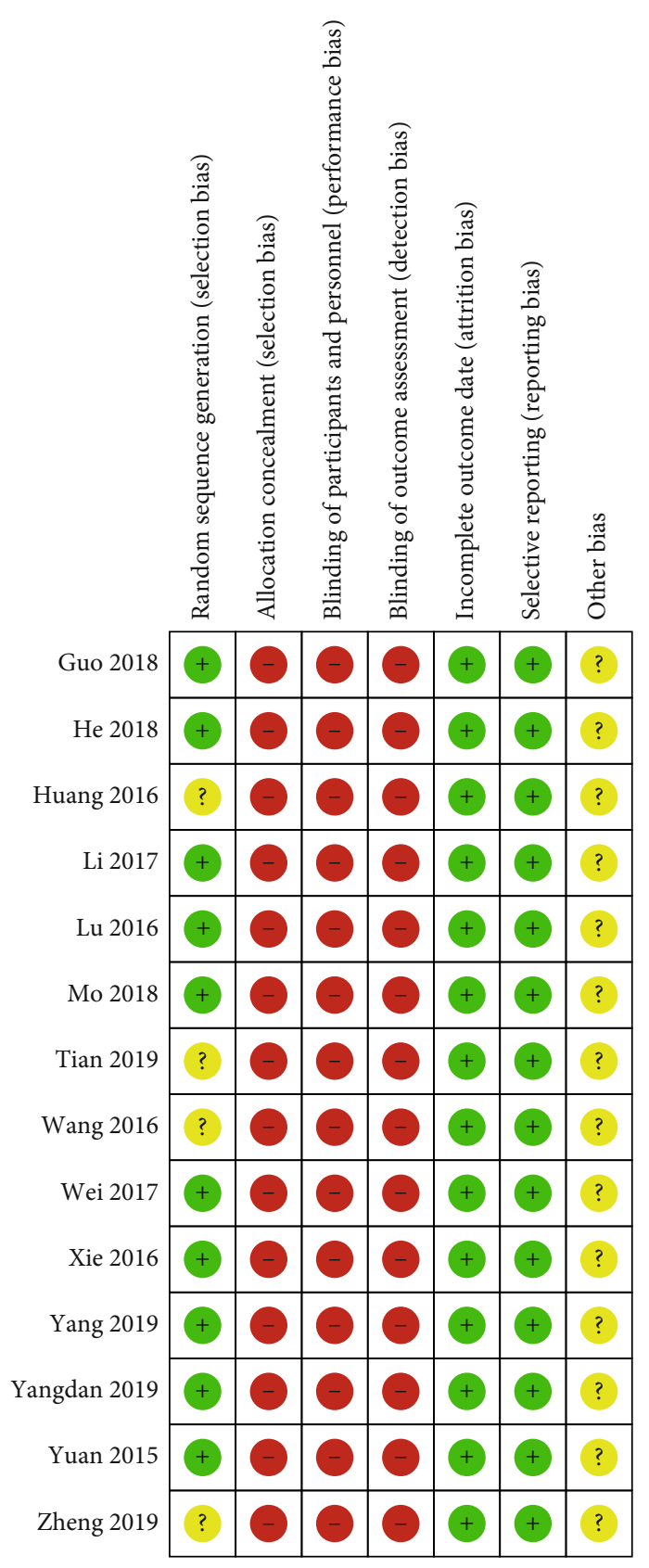

FIgURE 2: The risk of bias about each included study.

bias $(P=0.09)$. Sensitivity analysis was performed for the early pregnancy loss rate, and the effect estimate remained unchanged, which indicated the robustness of the pooled results (Figure 8).

4.4. GRADE Evaluation. We assessed the quality for the outcomes by GRADE evaluation. The quality of included studies was really low, and the "Risk of bias" was downgraded to "serious." The $I^{2}$ was high in the result of TCM syndromes and symptoms, and the "Inconsistency" was downgraded to "serious." The result of GRADE evaluation for TCM syndromes and symptoms was low, while the quality for other outcomes was moderate. The overall results of GRADE evaluation are summarized in Table 3. 


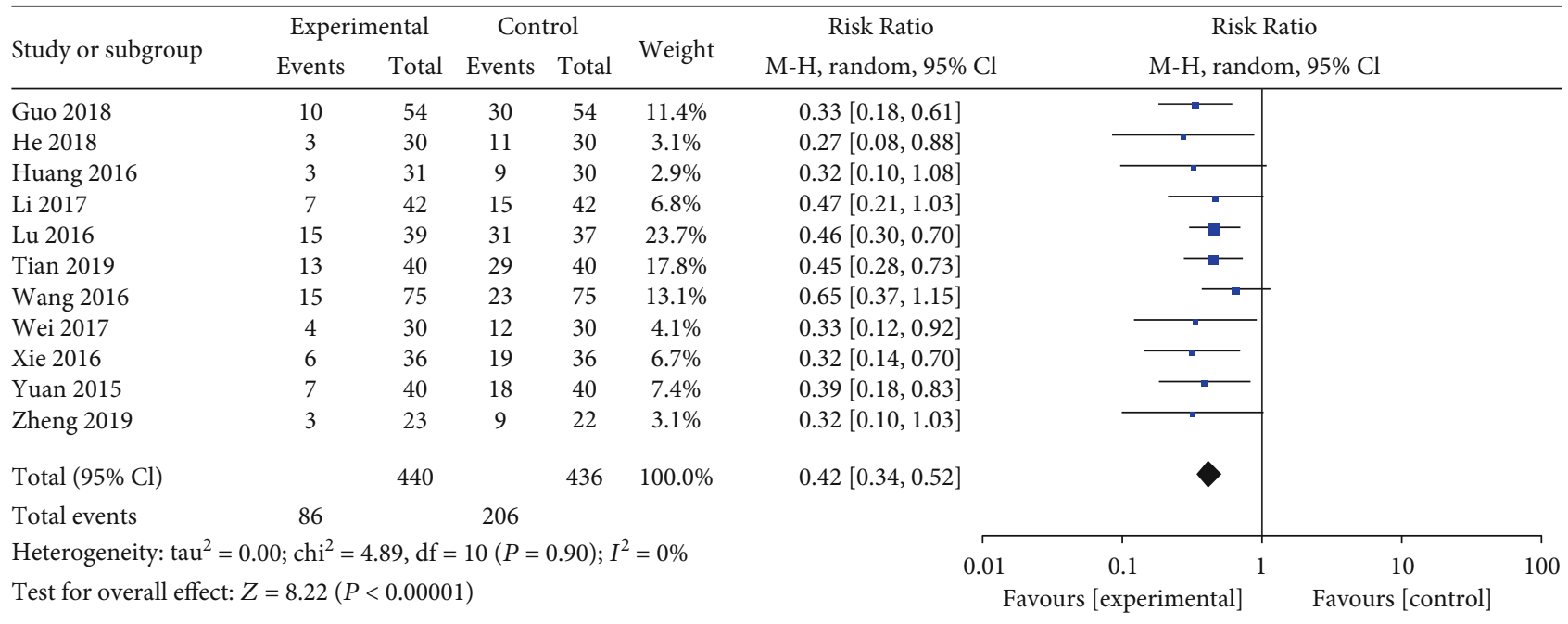

FIGURE 3: Forest plot for the incidence of early pregnancy loss between combined and western alone medicine group.

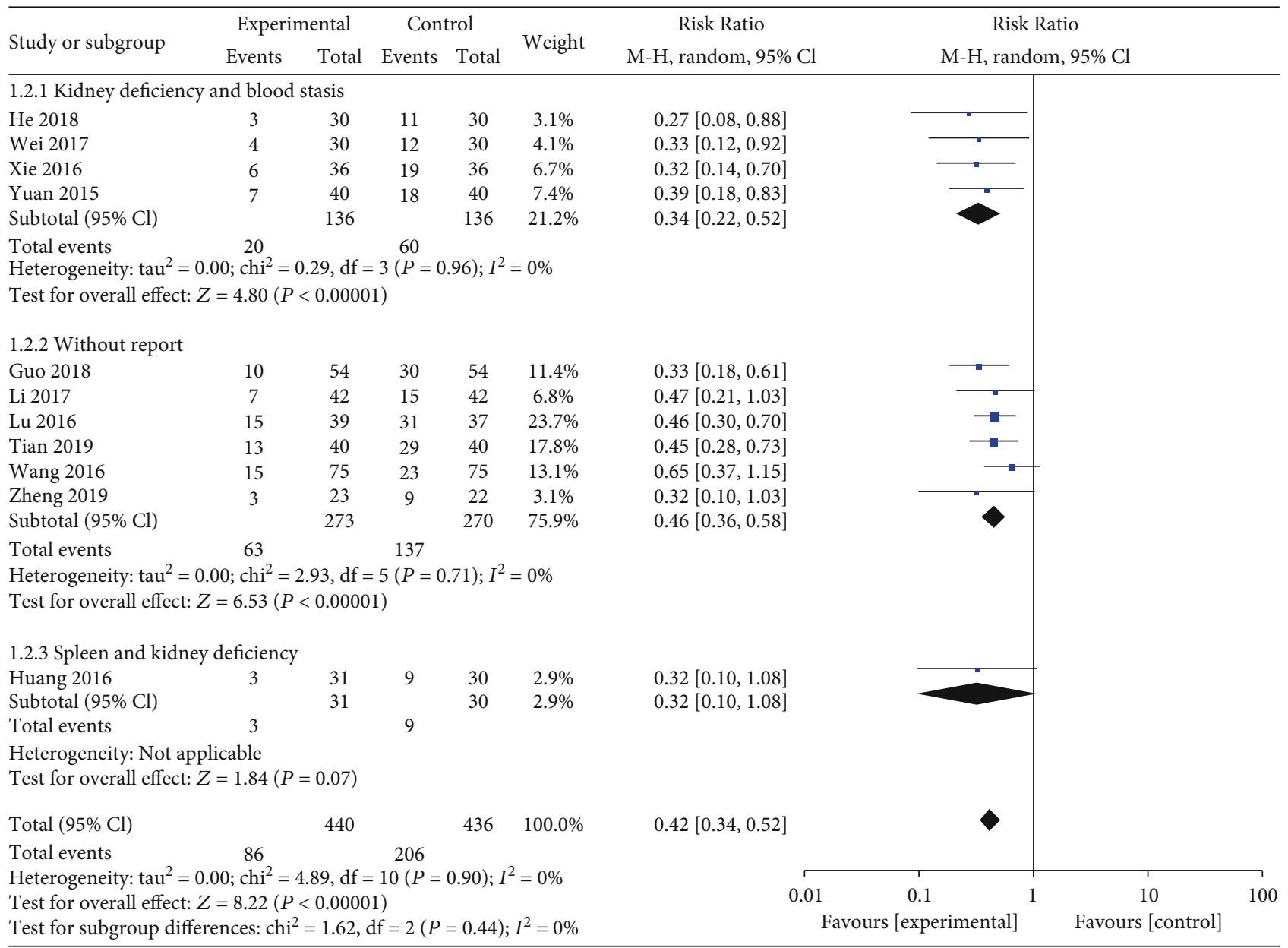

FIGURE 4: Subgroup analysis for early pregnancy loss rate based on the different types of TCM syndrome differentiation.

\section{Discussion}

5.1. Summary of Evidence. This meta-analysis demonstrated a quantitative estimates of the clinical efficacy and safety of cotreatment with STP and western medicine for the treat- ment of URSA by integrating outcomes from the 12 RCTs of 916 patients. The baseline of each study was consistent. Results from the meta-analysis showed that STP combined with western medicine can significantly reduce the early pregnancy loss incidence in the first trimester of pregnancy 


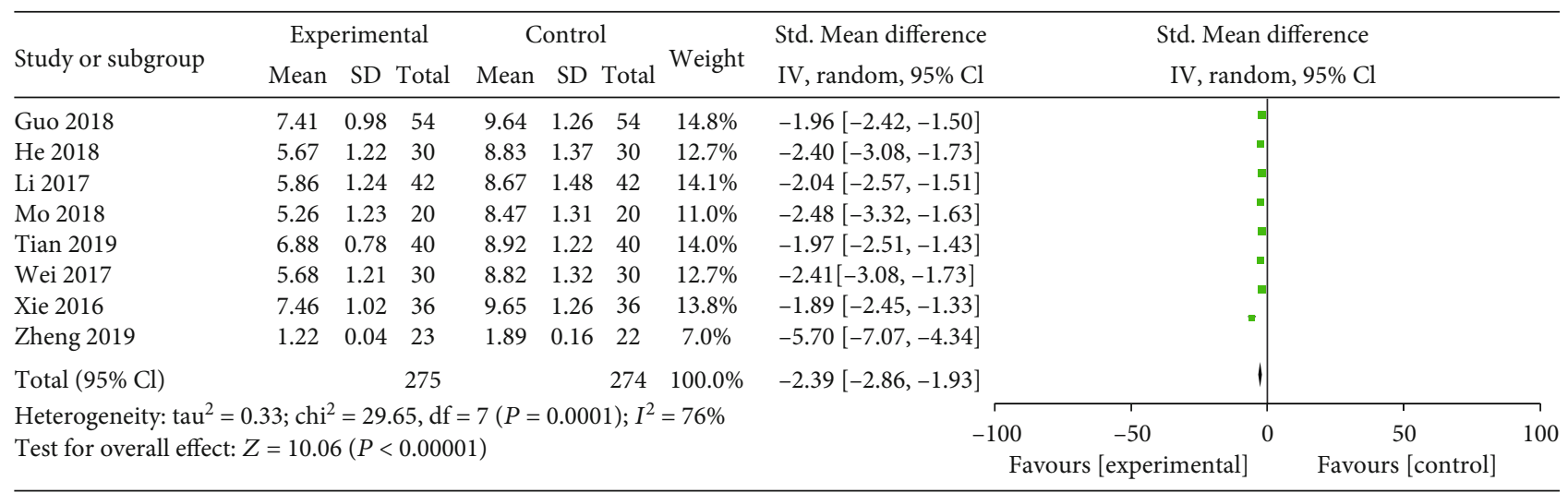

FIGURE 5: Forest plot for TCM syndromes and symptoms between combined and western alone medicine group.

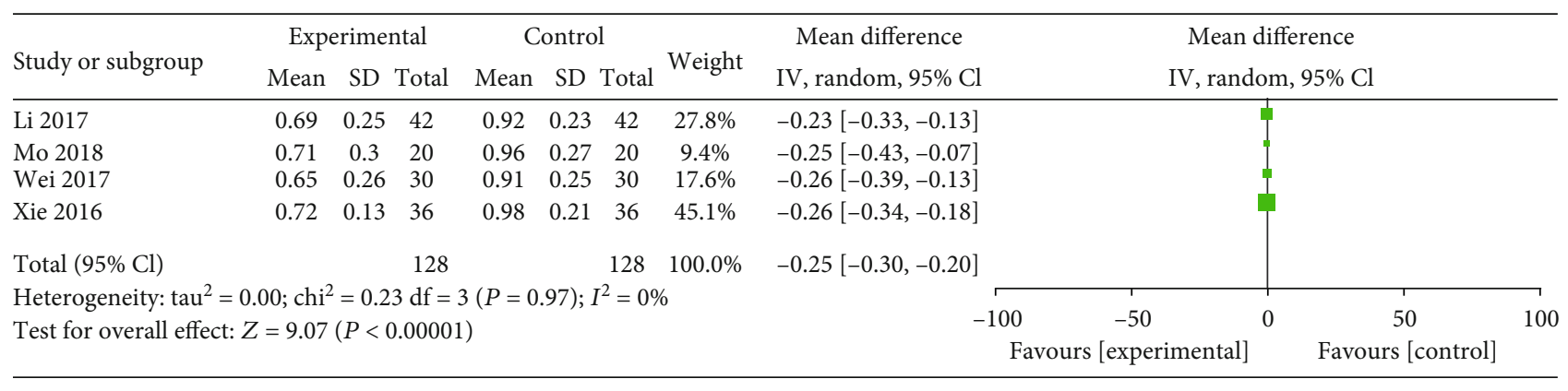

FIGURE 6: Forest plot for serum D-dimer level between combined and western alone medicine group.

\begin{tabular}{|c|c|c|c|c|c|c|c|c|c|}
\hline \multirow{2}{*}{ Study or subgroup } & \multicolumn{2}{|c|}{ Experimental } & \multicolumn{2}{|c|}{ Control } & \multirow{2}{*}{ Weight } & \multirow{2}{*}{$\begin{array}{c}\text { Risk Ratio } \\
\mathrm{M}-\mathrm{H}, \text { random, } 95 \% \mathrm{Cl}\end{array}$} & \multirow{2}{*}{\multicolumn{2}{|c|}{$\begin{array}{c}\text { Risk Ratio } \\
\mathrm{M}-\mathrm{H}, \text { random, } 95 \% \mathrm{Cl}\end{array}$}} & \\
\hline & Events & Total & Events & Total & & & & & \\
\hline Guo 2018 & 42 & 54 & 20 & 54 & $33.0 \%$ & $2.10[1.44,3.06]$ & & -- & \\
\hline He 2018 & 20 & 30 & 11 & 30 & $16.3 \%$ & $1.82[1.07,3.10]$ & & $=$ & \\
\hline Mo 2018 & 9 & 20 & 4 & 20 & $4.6 \%$ & $2.25[0.83,6.13]$ & & & \\
\hline Wei 2017 & 19 & 30 & 10 & 30 & $14.1 \%$ & $1.90[1.07,3.38]$ & & $\rightarrow$ & \\
\hline Zheng 2019 & 20 & 23 & 13 & 22 & $31.9 \%$ & $1.47[1.00,2.16]$ & & - & \\
\hline Total $(95 \% \mathrm{Cl})$ & & 157 & & 156 & $100.0 \%$ & $1.81[1.46,2.25]$ & & $>$ & \\
\hline Total events & 110 & & 58 & & & & & & \\
\hline \multirow{2}{*}{\multicolumn{6}{|c|}{$\begin{array}{l}\text { Heterogeneity: } \operatorname{tau}^{2}=0.00 ; \mathrm{chi}^{2}=2.05, \mathrm{df}=4(P=0.73) ; I^{2}=0 \% \\
\text { Test for overall effect: } Z=5.39(P<0.00001)\end{array}$}} & 0.01 & 0.1 & & 100 \\
\hline & & & & & & & experimental] & Favours & \\
\hline
\end{tabular}

FIGURE 7: Forest plot for live birth rate between combined and western alone medicine group.

in women with URSA compared to the control group. Our meta-analysis also indicated that, compared with control group, STP combined with western medicine has the more significant effect on the live birth rate, TCM syndromes and symptoms, and serum D-dimer level. The safety of STP for the treatment of URSA remains unclear. However, due to poor methodological quality, the conclusion about beneficial effect and safety of STP combined with western medicine needed to be further verified in future studies.

5.2. Promising Complementary Therapy for URSA. In recent years, the incidence of URSA has increased causing a considerable negative life occurrence and may cause notable physical and psychological damage to couples who attempt to have a child due to the multifactorial, complex, and poorly under- stood pathogenesis of URSA [44]. There is currently no universally accepted standard treatment for URSA owing to it being identified by a diagnosis of exclusion and its complex molecular and cellular regulation [2].

Whether progesterone as the major method used in most included studies, or immunologic intervention and anticoagulant therapy administrated in a few included studies, there tends to have controversy and limitations such as adverse reactions like dizziness, nausea, and vomiting; potential risks include allergies, infectious diseases, and bleeding along with increasing financial burden [10]. New approaches for URSA will be needed and available in the future; Chinese herbal medicines, which regulate human body as a whole based on the four principal diagnostic methods of inspection, auscultation and olfaction, inquiry, and pulse-taking, are of great 


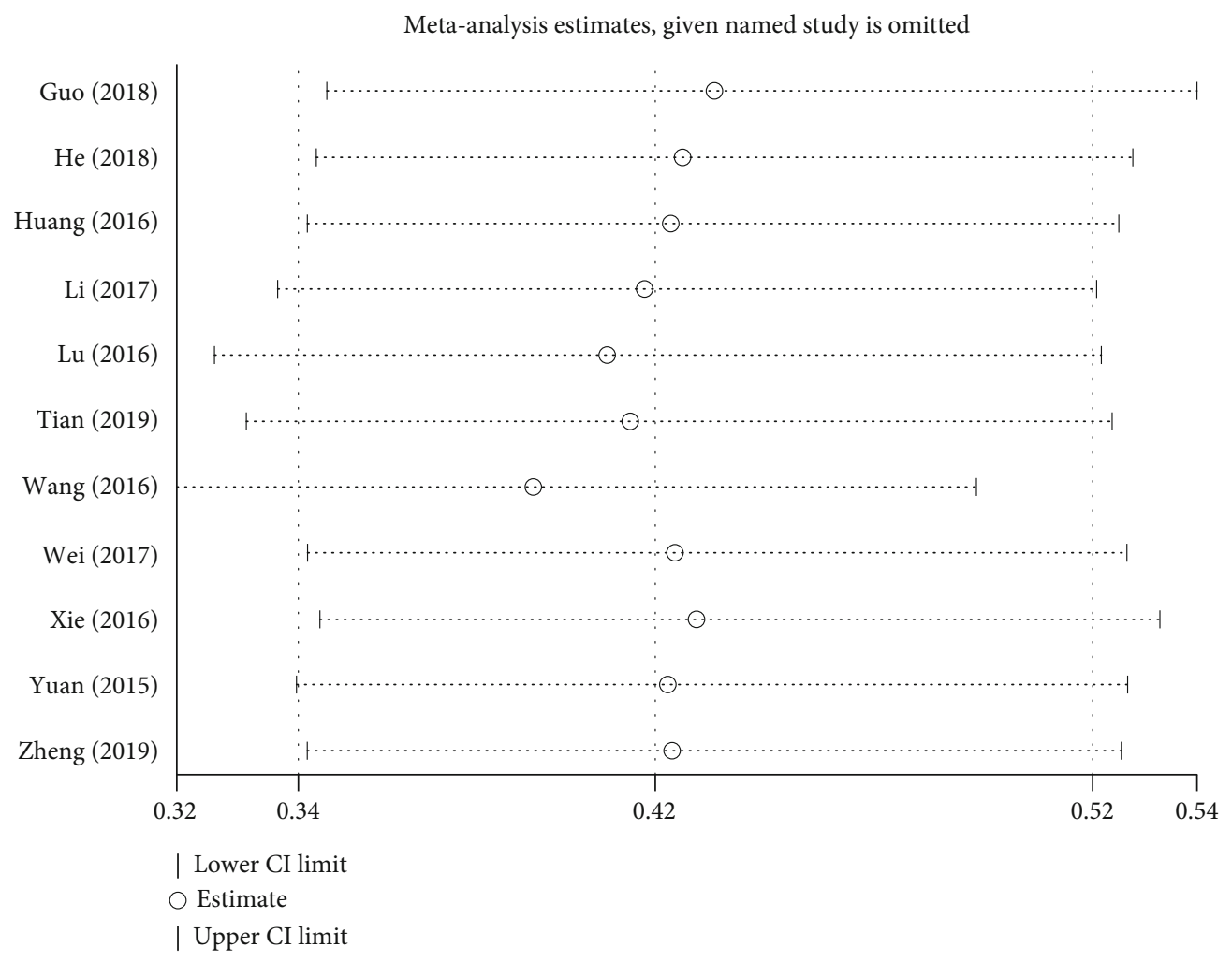

FIGURE 8: Sensitivity analysis for the early pregnancy loss rate.

TABLE 3: The summary of GRADE evaluation.

\begin{tabular}{|c|c|c|c|c|}
\hline Outcomes & Participants (studies) & RR/SMD (95\% CI) & GRADE & Comments \\
\hline $\begin{array}{l}\text { The incidence of early } \\
\text { pregnancy loss }\end{array}$ & 876 (11 RCTs) & $0.42(0.34,0.52)$ & $\bigoplus \oplus \bigoplus \bigcirc$ Moderate & $\begin{array}{l}\text { "Risk of bias" was downgraded to } \\
\text { "serious" }\end{array}$ \\
\hline $\begin{array}{l}\text { TCM syndromes and } \\
\text { symptoms }\end{array}$ & 549 (8 RCTs) & $-2.39(-2.86,-10.93)$ & $\bigoplus \oplus \bigcirc \bigcirc$ Low & $\begin{array}{l}\text { "Risk of bias" was downgraded to } \\
\text { "serious" } \\
\text { "Inconsistency" was downgraded to } \\
\text { "serious" }\end{array}$ \\
\hline Serum D-dimer level & 256 (4 RCTs) & $-0.25(-0.30,-0.20)$ & $\bigoplus \oplus \bigoplus \bigcirc$ Moderate & $\begin{array}{l}\text { "Risk of bias" was downgraded to } \\
\text { "serious" }\end{array}$ \\
\hline Live birth rate & 313 (5 RCTs) & $1.81(1.46,2.25)$ & $\bigoplus \bigoplus \bigoplus \bigcirc$ Moderate & $\begin{array}{l}\text { "Risk of bias" was downgraded to } \\
\text { "serious" }\end{array}$ \\
\hline
\end{tabular}

RR: Risk Ratio; SMD: Standard Mean Difference; RCT: Random controlled trial.

interest as complementary medicines [45]. Although it has the characteristics of complex composition and unclear mechanism, its comprehensive effect on disease, especially health care and prevention, is beyond the western medicine [46].

TCM has a unified medical theory for clinical diagnosis and treatment of URSA, which focuses on "Qi" and "blood" as the two basic elements of human physiology and believes that kidney-qi deficiency and blood stasis which can result in uterus pain and bleeding tendency are important in the pathology and mechanism of URSA [47]. Shoutai Pill, a well-known kidney-tonifying recipe in TCM for the prevention of miscarriage, is the basis of many other prescriptions [48]. In this meta-analysis, we found that STP combined with western medicine not only possess unique advantages in the prevention of miscarriage in patients with URSA but also has the potential to be highly effective at relieving symptoms and exhibiting good effects in activating blood flow and eliminating blood stasis by reducing the serum D-dimer level. It includes Chinese Dodder Seed as the main herb, supplemented by Chinese Taxillus Twig, Himalayan Teasel Root, and Donkey-hide Glue, playing the role of nourishing kidney and promoting generation of essence and blood to maintain a successful pregnancy preservation [28]. Among them, pharmacological studies have shown that the flavonoid components contained in Chinese Dodder Seed have estrogen-like function, which can improve reproductive endocrine function [49]; Alkaloids in Himalayan Teasel Root can inhibit uterine smooth muscle contraction and fight oxytocin [50]; flavonoid glycoside in Chinese Taxillus Twig has the action similar to progesterone which can supplement the insufficiency of 
endocrine function of patients [51]; and Donkey-hide Glue has a powerful function of enriching blood, exhibiting good effects in activating blood flow and eliminating blood stasis to significantly promote blood microcirculation [52], all of them affirm the important roles of Shoutai Pill in the treatment of URSA with no significant adverse effects found [53]. In summary, these herbal medicines can be used to improve the pregnancy outcome in the first trimester of pregnancy in women with URSA. Animal experiments reported that its treatment roles in the maintenance of normal pregnancy and in the mechanism of reducing adverse pregnancy outcomes may have multiple targets related to the regulation of immune conditions [54], the reduction of the apoptosis of trophoblast cells [55], and the improvement of hypothalamus-pituitarygonadal axis [56]. However, valid evidence, including metaanalysis results, has yet to be needed for further recommendation.

\section{Limitations}

Although, we have comprehensively analyzed and evaluated all studies, it still has limitations that should be acknowledged. First, the included studies had low quality due to an unclear allocation concealment, selective bias, attrition bias, and blinding methods. Second, although we conducted an unbiased literature search without language restriction, all trials included in this review were conducted in China and were published in Chinese without relevant foreign experiments, which likely lead to a potential bias and therefore limit their representativeness. Third, few studies included have mentioned the adverse reactions during and after treatment. Meanwhile, no trial reported long-term follow-up, so the long-term safety of the intervention is still not well known. Fourth, the criteria for the outcome of each study were inconsistent. As a result, the evaluation had certain subjectivity and difference, which affected the accuracy and stability of the test.

\section{Conclusion}

In summary, our results showed that Shoutai Pill in combination with western medicine might increase the chances of a successful pregnancy in the first trimester of pregnancy for unexplained recurrent spontaneous abortion and may be beneficial for women with URSA as adjunct therapies without obvious adverse event in the experimental group. However, due to the relatively low quality of the included studies, we should be more cautious to promote this result. Future well-designed, multicenter, and large-sample clinical studies on evaluating the efficacy and safety of STP are needed to ensure the scientific, objective, and reliable conclusions of the research so as to make the results more convincing and provide more valuable information.

\section{Data Availability}

The data set supporting the results of this article is included within the article.

\section{Conflicts of Interest}

The authors declare that there are no conflicts of interest regarding the publication of this paper.

\section{Acknowledgments}

This study was supported by grants from Jiaxing Key DISCIPLINE of Traditional Chinese Medicine-Integrated Chinese and Western Medicine Gynecology (2019-2021) and Jiaxing Key DISCIPLINE of Medicine-Anesthesiology (2019-zc-06).

\section{References}

[1] Practice Committee of the American Society for Reproductive Medicine, "Evaluation and treatment of recurrent pregnancy loss: a committee opinion," Fertility and Sterility, vol. 98, no. 5, pp. 1103-1111, 2012.

[2] T. C. Li, M. Makris, M. Tomsu, E. Tuckerman, and S. Laird, "Recurrent miscarriage: aetiology, management and prognosis," Human Reproduction Update, vol. 8, no. 5, pp. 463-481, 2002.

[3] C. Garrido-Gimenez and J. Alijotas-Reig, "Recurrent miscarriage: causes, evaluation and management," Postgraduate Medical Journal, vol. 91, no. 1073, pp. 151-162, 2015.

[4] R. Rai and L. Regan, "Recurrent miscarriage," The Lancet, vol. 368, no. 9535, pp. 601-611, 2006.

[5] N. Pereza, S. Ostojić, M. Kapović, and B. Peterlin, "Systematic review and meta-analysis of genetic association studies in idiopathic recurrent spontaneous abortion," Fertility and Sterility, vol. 107, no. 1, pp. 150-159.e2, 2017.

[6] W. Qin, Y. Tang, N. Yang, X. Wei, and J. Wu, "Potential role of circulating microRNAs as a biomarker for unexplained recurrent spontaneous abortion," Fertility and Sterility, vol. 105, no. 5, pp. 1247-1254.e3, 2016.

[7] S. Hosseini, F. Shokri, S. A. Pour, J. Khoshnoodi, M. JeddiTehrani, and A. H. Zarnani, "Diminished frequency of menstrual and peripheral blood NKT-like cells in patients with unexplained recurrent spontaneous abortion and infertile women," Reproductive Sciences, vol. 26, no. 1, pp. 97-108, 2017.

[8] P. Wang, H. Yang, G. Wang, and J. Tian, "Predictive value of thromboelastography parameters combined with antithrombin III and D-Dimer in patients with recurrent spontaneous abortion," American Journal of Reproductive Immunology, vol. 82, no. 4, p. e13165, 2019.

[9] Z. Liu, H. Xu, X. Kang, T. Wang, L. He, and A. Zhao, "Allogenic lymphocyte immunotherapy for unexplained recurrent spontaneous abortion: a meta-analysis," American Journal of Reproductive Immunology, vol. 76, no. 6, pp. 443-453, 2016.

[10] S. W. Wang, S. Y. Zhong, L. J. Lou, Z. F. Hu, H. Y. Sun, and H. Y. Zhu, "The effect of intravenous immunoglobulin passive immunotherapy on unexplained recurrent spontaneous abortion: a meta-analysis," Reproductive Biomedicine Online, vol. 33, no. 6, pp. 720-736, 2016.

[11] H. Ou and Q. Yu, "Efficacy of aspirin, prednisone, and multivitamin triple therapy in treating unexplained recurrent spontaneous abortion: a cohort study," International Journal of Gynaecology and Obstetrics, vol. 148, no. 1, pp. 21-26, 2020.

[12] G. L. Xu, X. F. Hu, Y. M. Han, and A. W. Wei, "Clinical efficacy of low molecular heparin on unexplained recurrent 
spontaneous abortion," Clinical Laboratory, vol. 64, no. 6, pp. 1037-1040, 2018.

[13] G. Saccone, C. Schoen, J. M. Franasiak, R. T. Scott Jr., and V. Berghella, "Supplementation with progestogens in the first trimester of pregnancy to prevent miscarriage in women with unexplained recurrent miscarriage: a systematic review and meta-analysis of randomized, controlled trials," Fertility and Sterility, vol. 107, no. 2, pp. 430-438.e3, 2017.

[14] M. Tavakoli, M. Jeddi-Tehrani, A. Salek-Moghaddam et al., "Effects of 1,25(OH)2 vitamin D3 on cytokine production by endometrial cells of women with recurrent spontaneous abortion," Fertility and Sterility, vol. 96, no. 3, pp. 751-757, 2011.

[15] A. Hekmatdoost, F. Vahid, Z. Yari et al., "Methyltetrahydrofolate vs folic acid supplementation in idiopathic recurrent miscarriage with respect to methylenetetrahydrofolate reductase C677T and A1298C polymorphisms: a randomized controlled trial," PLoS One, vol. 10, no. 12, p. e0143569, 2015.

[16] N. Jia and J. Li, "Noncoding RNAs in unexplained recurrent spontaneous abortions and their diagnostic potential," Disease Markers, vol. 2019, Article ID 7090767, 7 pages, 2019.

[17] X. Cao, Y. Cui, X. Zhang, J. Lou, J. Zhou, and R. Wei, “The correlation of sperm morphology with unexplained recurrent spontaneous abortion: a systematic review and meta-analysis," Oncotarget, vol. 8, no. 33, pp. 55646-55656, 2017.

[18] Z. Tavoli, M. Mohammadi, A. Tavoli et al., "Quality of life and psychological distress in women with recurrent miscarriage: a comparative study," Health Qual Life Outcomes, vol. 16, no. 1, p. 150, 2018.

[19] M. M. Wagner, M. M. Beshay, S. Rooijakkers et al., "Increased cardiovascular disease risk in women with a history of recurrent miscarriage," Acta Obstetricia et Gynecologica Scandinavica, vol. 97, no. 10, pp. 1192-1199, 2018.

[20] S. L. Yang, T. T. Niu, X. L. Li, D. J. Li, M. Q. Li, and H. Y. Wang, "Bu-Shen-Yi-Qi formula impairs cytotoxicity of NK cells by up-regulating IDO expression in trophoblasts," Gynecological Endocrinology, vol. 34, no. 8, pp. 675-679, 2018.

[21] G.-Y. Yang, H. Luo, X. Liao, and J.-P. Liu, "Chinese herbal medicine for the treatment of recurrent miscarriage: a systematic review of randomized clinical trials," BMC Complementary and Alternative Medicine, vol. 13, no. 1, p. 320, 2013.

[22] L. Li, L. Dou, P. C. Leung, T. K. H. Chung, C. C. Wang, and Cochrane Pregnancy and Childbirth Group, "Chinese herbal medicines for unexplained recurrent miscarriage," Cochrane Database of Systematic Reviews, vol. 14, no. 1, article Cd010568, 2016.

[23] X. L. Feng, Z. Chang, X. X. Zhao, W. Wang, W. W. Li, and L. Chen, "Prevalence of type D personality and distribution of TCM syndromes in unexplained recurrent Spontane abortion patients," Chinese General Practice, vol. 21, no. 34, pp. 4227-4231, 2018.

[24] X. Li, Q. M. Zhang, S. P. Liang et al., "Biological basis of kidney governing reproduction," Acta Chinese Medicine, vol. 35, no. 1, pp. 14-18, 2020.

[25] T. C. Cao, Q. B. Lu, and C. Chen, "Research Progress of Bushen Huoxue Chinese medicine in preventing and treating immunological recurrent spontaneous abortion," Acta Chinese Medicine, vol. 34, no. 12, pp. 2554-2557, 2019.

[26] S. Jiang, Y. Zhang, L. Chen et al., "Effect of method of Tonifying kidney and activating blood circulation regulating progesterone on deciduating of URSA in late secretory phase,"
Chinese Archives of Traditional Chinese Medicine, vol. 37, no. 10, pp. 2362-2368, 2019.

[27] J. Zhang, L. Chen, C. H. Zheng, J. Wang, D. Xie, and Y. X. Zhou, "Effect of Shoutai pills on Th1/Th2 cytokines in serum and endometrium of rats with stimulated ovulation," Current Medical Science, vol. 39, no. 2, pp. 285-290, 2019.

[28] L. Zhao, Y. Xu, X. Duo, X. Tian, and P. Wang, "A probe into effect of Shoutai pill on endometrial receptivity," Experimental and Therapeutic Medicine, vol. 15, no. 4, pp. 3765-3768, 2018.

[29] Y. Q. Feng, "Clinical effects of Shoutai Pills combined with Antai Fuyuan Drink on patients with unexplained unexplained recurrent spontaneous abortion due to kidney deficiency and blood stasis pattern," Chinese Traditional Patent Medicine, vol. 41, no. 12, pp. 2908-2913, 2019.

[30] C. C. Liang, L. Lei, S. S. Mao, and S. Zhang, "Study on effect of Shoutai Pills on aquaporin expression in uterine decidua of recurrent abortion rats," Chinese Archives of Traditional Chinese Medicine, vol. 37, no. 11, pp. 2657-2661, 2019.

[31] D. Moher, A. Liberati, J. Tetzlaff, D. G. Altman, and PRISMA Group, "Preferred reporting items for systematic reviews and meta-analyses: the PRISMA statement," Journal of Clinical Epidemiology, vol. 62, no. 10, pp. 1006-1012, 2009.

[32] M. Guo, "Clinical efficacy of modified Shoutai Pill combined with progesterone in the treatment of recurrent spontaneous abortion," Journal of Hubei Minzu University (Medical Edition), vol. 35, no. 3, pp. 7-9, 2018.

[33] D. H. He, "Observation on the effect of Shoutai Pill and Siwu Decoction combined with progesterone on recurrent spontaneous abortion with kidney deficiency and blood stasis," Journal of Practical Traditional Chinese Medicine, vol. 34, no. 4, pp. 458-459, 2018.

[34] C. S. Huang, Y. C. Guan, and X. Y. Liu, "Treatment of 31 cases of recurrent spontaneous abortion with deficiency of spleen and kidney by integrated traditional Chinese and Western medicine," Jiangxi Journal of Traditional Chinese Medicine, vol. 47, no. 4, pp. 50-52, 2016.

[35] L. J. Li, H. Liang, A. L. Wang, J. F. Fu, and Y. Q. Hao, "Effect of Shoutai Pill and Siwu Decoction combined with progesterone tablet on the immune factors of patients with unexplained recurrent spontaneous abortion," World Chinese Medicine, vol. 12, no. 11, pp. 2713-2715+2719, 2017.

[36] T. Lu, "Clinical efficacy of Shoutai Pill combined with didrogesterone on the immune factors of patients with unexplained recurrent spontaneous abortion," Journal of New Chinese Medicine, vol. 48, no. 8, pp. 182-184, 2016.

[37] P. H. Mo, D. Q. Lan, and Z. Q. Yan, "Effect of modified Shoutai Pill combined with Siwu Decoction on immune factors in recurrent abortion patients with kidney deficiency and blood stasis," Liaoning Journal of Traditional Chinese Medicine, vol. 45, no. 6, pp. 1205-1207, 2018.

[38] H. Tian and D. M. He, "Clinical efficacy of Shoutai Pill combined with didrogesterone on recurrent abortion," Shenzhen Journal of Integrated Traditional Chinese and Western Medicine, vol. 29, no. 10, pp. 41-42, 2019.

[39] J. N. Wei, A. L. Wang, Y. Chen, and Y. X. Chen, "Effect of Shoutai Pill and Siwu Decoction on immune factors of patients with kidney deficiency and blood stasis type of recurrent spontaneous abortion," Liaoning Journal of Traditional Chinese Medicine, vol. 44, no. 9, pp. 1887-1889, 2017.

[40] Y. H. Xie and X. Yang, "Observation on the clinical effect of Shoutai Pill combined with Siwu Decoction and 
Didrogesterone in the treatment of recurrent abortion," Journal of New Chinese Medicine, vol. 48, no. 6, pp. 156-158, 2016.

[41] Y. Yuan and Y. Wang, "The clinical effect and influence on TNF- $\alpha$, IL-6, IL-10 of Shoutai Pill combined with dydrogesterone on recurrent abortion," Guiding Journal of Traditional Chinese Medicine and Pharmacy, vol. 21, no. 23, p. 69, 2015.

[42] Y. Wang and Q. X. Sun, "Effect of integrated traditional Chinese and Western medicine on Th1 / Th2 cytokines in unexplained recurrent abortion patients," Modern Journal of Integrated Traditional Chinese and Western Medicine, vol. 25, no. 14, pp. 1526-1528, 2016.

[43] X. J. Zheng, "The clinical effect of Shoutai Pill combined with progesterone, didroxyprogesterone and low molecular weight heparin sodium on recurrent abortion of kidney deficiency type," Chinese and Foreign Medical Research, vol. 17, no. 11, pp. 149-151, 2019.

[44] E. Toffol, P. Koponen, and T. Partonen, "Miscarriage and mental health: results of two population-based studies," Psychiatry Research, vol. 205, no. 1-2, pp. 151-158, 2013.

[45] F. Liu and S. P. Luo, "Effect of Chinese herbal treatment on Th1- and Th2-type cytokines, progesterone and beta-human chorionic gonadotropin in early pregnant women of threatened abortion," Chinese Journal of Integrative Medicine, vol. 15, no. 5, pp. 353-358, 2009.

[46] B. R. Martin, "Complementary medicine therapies that may assist with weight loss: a narrative review," Journal of Chiropractic Medicine, vol. 18, no. 2, pp. 115-126, 2019.

[47] L. Che, Y. Wang, Q. X. Fang, and R. X. Chen, "Clinical thinking of TCM in treating recurrent spontaneous abortion," Chinese Journal of Information on Traditional Chinese Medicine, vol. 27, no. 2, pp. 99-101, 2020.

[48] J. Gao and S. P. Luo, "Kidney-tonifying and abortion preventing effects of Shou Tai Wan by different extration methods on rats," Journal of Chinese Medicinal Materials, vol. 34, no. 8, pp. 1251-1255, 2011.

[49] H. W. Wu, Y. H. Feng, D. Y. Wang et al., "Effect of total flavones from Cuscuta Chinensis on anti-abortion via the MAPK signaling pathway," Evidence-Based Complementary and Alternative Medicine, vol. 2018, no. 2018, Article ID 6356190, 12 pages, 2018.

[50] E. W. Liu, S. Wu, and G. W. Fan, "Chemical constituents and pharmacologic actions of the plants from Dipsacus asperoides," Chinese Archives of Traditional Chinese Medicine, vol. 28, no. 7, pp. 1421-1423, 2010.

[51] Y. J. Liu, G. Y. Huang, M. W. Yang, P. Gong, and F. Lu, "Effect of jiantai liquid on expression of estrogen and progesterone receptors in uterus of mice with embryo implantation dysfunction induced by mifepristone," Chinese Journal of Integrated Traditional and Western Medicine, vol. 24, no. 9, pp. 816819, 2004.

[52] Y. B. Du, H. R. Fan, and Z. Yan, "Advances in chemical composition and pharmacological action of donkey-hide glue," Journal of Tianjin Medical University, vol. 24, no. 3, pp. 267270, 2018.

[53] G. Peng, Reproductive safety of Shoutai Pill on gestation of sd rats and embryo developmen, [M.S. thesis], Hunan University Of Traditional Chinese Medicine, Hunan, China, 2009.

[54] M. Lai, Z. You, H. Ma et al., "Effects of Shoutai pills on expression of Th1/Th2 cytokine in maternal-fetal interface and pregnancy outcome," China Journal of Chinese Materia Medica, vol. 35, no. 22, pp. 3065-3068, 2010.
[55] Z. W. Tan, L. Lei, L. Luo, and L. L. Lu, "Effects of Shoutai Wan on proteome in decidua tissues of recurrent abortion mice," China Journal of Chinese Materia Medica, vol. 38, no. 4, pp. 591-596, 2013.

[56] D. N. Qin, B. R. She, Y. C. She, and J. H. Wang, "Effects of flavonoids from Semen Cuscutae on the reproductive system in male rats," Asian Journal of Andrology, vol. 2, no. 2, pp. 99 $102,2000$. 